\title{
Model for the Whole Roller Leveling Process of Plates with Random Curvature Distribution Based on the Curvature Integration Method
}

\author{
Ben Guan ${ }^{1,2}$, Chao Zhang ${ }^{1}$, Yong Zang ${ }^{1 *}$ and Yuan Wang ${ }^{1}$
}

\begin{abstract}
A model based on the curvature integration method has been applied in an online plate leveling system. However, there are some shortcomings in the current leveling models. On the one hand, the models cannot deal with the leveling process of plates with a random curvature distribution. On the other hand, the current models are suitable only for stable leveling processes and ignore the biting in and tailing out stages. This study presents a new plate-leveling model based on the curvature integration method, which can describe the leveling process of plates with random curvature distribution. Further, the model is solved in two cases in order to take the biting in and tailing out stages into consideration. The proposed model is evaluated by comparing with a plate leveling experiment. Finally, the leveling process of a plate with a wave bent is studied using the proposed model. It is found that the contact angles vary greatly during the biting in and tailing out stages. However, they are relatively steady during the 5 roller leveling stage. In addition, the contact angle of roller No. 2 is the smallest, which is close to 0 . Roller leveling can effectively eliminate bending in the plate, but there are regions in the head and tail of the plate, where roller leveling is not effective. The non-leveling region length is about 2 times that of the roller space. This study proposes a quasi-static plate-leveling model, which makes it possible to analyze the dynamic straightening process using a curvature integration method. It also makes it possible to analyze the straightening process of a plate with random curvature distribution.
\end{abstract}

Keywords: Leveling, Curvature integration method, Curvature, Contact angle, Deflection

\section{Introduction}

A plate is usually subjected to bending deformation during rolling, cooling, transportation, and other processes owing to external forces, temperature changes, and internal forces. Thus, the leveling process is indispensable for flat products. Leveling can effectively eliminate uneven deformation in a plate. Further, residual stress distribution in the plate can also be improved for its further use [1]. The roller leveling process has been widely used in the continuous production of sheet metal thanks to its high efficiency and high effectiveness [2]. In order to meet the increasingly stringent requirements, plate leveling research is focused on developing more accurate

\footnotetext{
*Correspondence: yzang@ustb.edu.cn

${ }^{1}$ School of Mechanical Engineering, University of Science

and Technology Beijing, Beijing 100083, China

Full list of author information is available at the end of the article
}

leveling models that can fully utilize latest detection and control technology [3].

An effective leveling strategy depends on an accurate analysis of the leveling process, which requires the establishment of a reasonable analytical model. Based on literature survey, analytical models on plate leveling can mainly be divided into three categories. In the first category, leveling analytical models are developed based on the beam bending theory, where the leveling process is studied under an assumption of multi-section and continuous beams [4-11]. This methodology has been widely used to analyze plate and profile leveling processes and some theoretical achievements could be obtained. Zang et al. [12] reported the analytical solution of H-beam bending deflection in the leveling process with nine rollers. Guan et al. [13] studied stress-inheriting behavior during leveling and its influence on the leveling process. Zhou [14] established an analytical model for the roller 
bending process, where the total deformation curvature is regarded as an independent variable. Liu et al. [15] studied the evolution of stress in a continuous bending process and the effect of the strain hardening model was also studied. Despite being widely available, these models fail to satisfy the ever-increasing requirement for flatness, because the models do not fully consider the contact state between the plate and rollers. The second category consists of finite element method (FEM)-based and boundary element method (BEM)-based analytical models [16-20]. Huh et al. [21] studied the sheet tension leveling process with the help of elastic-plastic finite element simulations. Mathieu et al. [22] simulated a sheet threading process using bridle rolls. Yoon et al. [23] simulated the tension leveling process using $2 \mathrm{D}$ and $3 \mathrm{D}$ frameworks. It was concluded that 3D simulations should be used for the tension leveling process. The residual stress in a UIC-60 rail after leveling was investigated by Betego'n et al. [24] using 1D, 2D, and 3D simulation models. However, FEM-based analysis of the leveling process is generally time-consuming, which restricts its use in industrial applications. The third category includes the curvature integration method. In this method, a number of iterations are needed to obtain the curvature evolution of the plate in question during the progressive leveling process, in which a relationship between curvature distribution and reduction of rollers is established. Using this method, Kadota et al. [25] studied the leveling process for different materials considering a variable set of process parameters. Instead of the traditional bilinear hardening model, Higo et al. [26] carefully considered the transition behavior of the selected material from an elastic to plastic state and made use of the curvature integration method, consequently improving the prediction of curvature evolution during the leveling process. Xue [27] improved the geometry characterization of the curvature integration method and derived the recurrence formulae of contact angles. Liu et al. [28] studied stress distribution along the width direction. Li et al. [29] studied the effect of an inter-mesh on the residual curvature of a longitudinal profile plate. Thanks to its high accuracy and efficiency, the curvature integration method is preferred for engineering applications. Based on the above analysis, the curvature integration method is selected for the establishment of a target model in this study.

In the current curvature integration models, a plate is usually considered to be bent with a large single curvature during the leveling process. The assumption will inevitably result in an imprecise curvature prediction of the leveled plate. Simultaneously, curvature is generally simplified in the calculation process, where the denominator of the curvature formula is replaced by 1 . This simplification causes small errors in solving the curvature.
However, an accumulative error is caused when building the relationship between curvature and deflection. Further, the models are effective only for stable leveling stages, because they do not take into consideration the biting in and tailing out stages. The biting in stage will affect the subsequent leveling stage; both ends of the plate are related to the biting in and tailing out stages. Therefore, it is necessary to take biting in and tailing out stages into consideration. Thanks to developments in detection technology, real-time online flatness information of a plate can be obtained. Based on these considerations, this paper presents an online precision leveling model, which is suitable for the current detection technology.

In this study, firstly, the initial deflection curve of a plate is described by a certain function. The curve is discretized into elements of equal length along the longitudinal direction. The accumulated error caused by curvature simplification is discussed in detail. Secondly, a curvature integration model with a high accuracy is established, which can describe the leveling process of a plate with random curvature distribution. To take the biting in and tailing out stages into consideration in addition to the stable leveling stage, the model is solved with contact establishment, and dynamic leveling is considered. Later, the analytical model is experimentally verified. Finally, the proposed model is used to analyze the plate roller leveling process.

\section{Curvature Integration Model for the Plate Roller Leveling Process}

\subsection{Curvature Integration Geometry Model}

The initial deflection curve of plate is defined by $f(x)$. Its first derivative $g(x)$ and the second derivative $k(x)$ are as follows:

$$
\left\{\begin{array}{l}
g(x)=f^{\prime}(x) \\
k(x)=g^{\prime}(x)
\end{array}\right.
$$

where $x \in[0, L]$ and $L$ is the plate length.

The deflection curve is evenly dispersed into several elements, as shown in Figure 1. The length of the elements along the longitudinal direction is $\Delta x$. As the

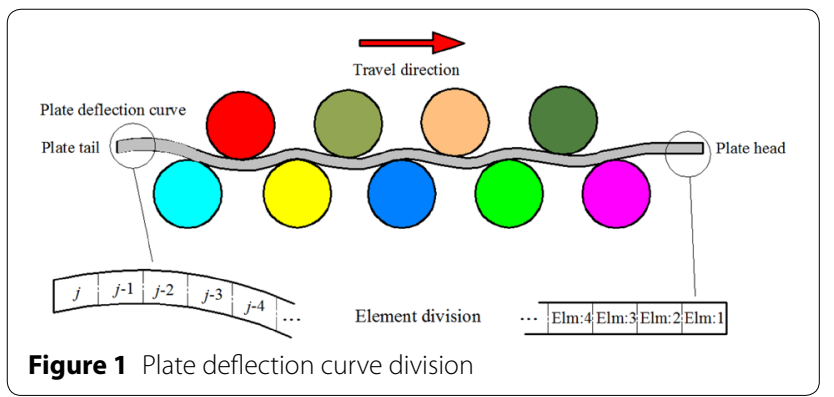


length of the elements is very small, the first derivative and second derivative of the elements on the initial deflection curve can be replaced by the first derivative and second derivative of any point on the elements, respectively. In the current study, the plate end that first enters into the rollers is named as the head and the other end is named as the tail. The elements are numbered from head to tail, as shown in Figure 1. For the element $j$, Eq. (1) can be rewritten as follows:

$$
\left\{\begin{array}{l}
g(j)=g(j \Delta x) \\
k(j)=k(j \Delta x)
\end{array}\right.
$$

Consequently, the curvature of element $j$ is as follows:

$$
A(j)=\frac{\left|f^{\prime \prime}\right|}{\left(1+f^{\prime 2}\right)^{3 / 2}}=\frac{|k(j \Delta x)|}{\left(1+(g(j \Delta x))^{2}\right)^{3 / 2}} .
$$

In the bending process with a small deflection, the denominator of Eq. (3) is generally replaced by 1 in engineering applications, because the item $(g(\Delta x j))^{2}$ is far less than 1. Thus, Eq. (3) can be simplified into the following equation:

$$
A(j)=|k(j \Delta x)| .
$$

In the existing curvature integration models, the above simplification is generally adopted in the establishment of a relationship between the curvature and deflection. The basic idea is to establish a geometry model for the first integral and the second integral of the curvature along the plate length direction. However, the above simplification will cause an accumulative error in the deflection curve, which is calculated by curvature distribution. Assuming that there is a curvature error of $\Delta A$ in the first element located at the head of the plate during the solving procedure, it will cause a deflection error of $\int_{0}^{L} \int_{0}^{x} g(1) \triangle A \mathrm{~d} x \mathrm{~d} X$ at the element of the plate tail, namely, $g(1) \triangle A L^{2} / 2$. The error caused by simplification for each element will obviously reduce the solving accuracy of the plate deflection curve, especially in the case of a large curvature deflection. Further, existing models consider the dip angle instead of the slope when building a relationship between slope and deflection, which also causes accumulative error. Therefore, it is necessary to adopt a curvature with less error during the establishment of a curvature integration model.

As the thickness of the plate during leveling remains constant, the deformation in the plate can be described by a plate neutral layer curve. The geometry model of the leveling process is shown in Figure 2.

Two adjacent rollers together are considered as a leveling span. For leveling with $n$ rollers, the process can be divided into $n-1$ leveling spans. The local coordinate system is set up at the contact point between the plate

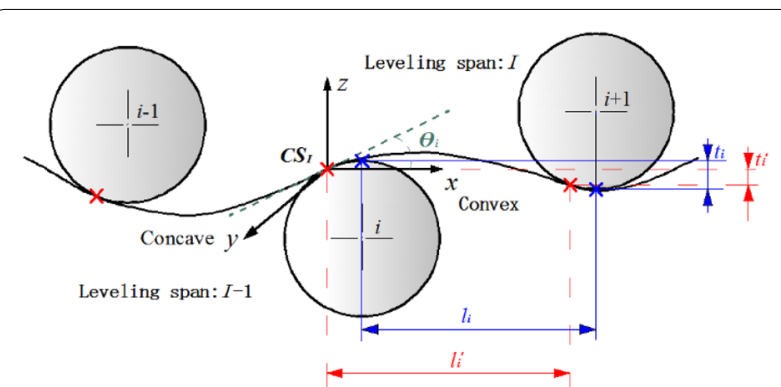

Figure 2 Plate roller leveling process diagram

and roller and the corresponding positive direction of the $x$-axis coincides with the leveling direction of the plate, as shown in Figure 2. The curvature is considered positive when the curve is concave. Correspondingly, the curvature is negative when the curve is convex. In a similar manner, the moment making the plate bend in a concave fashion is defined as positive and the moment responsible for the convex bending of the plate as negative. $R$ is the roller radius. As the plate is replaced by its neutral layer, the roller radius $r$ used in the calculation is the sum of the actual roller radius and half thickness of the plate. $L_{i}$ is the half length of the roller space, $l_{i}^{\prime}$ is the distance between adjacent contact points along the leveling direction, $t_{i}$ is the inter-mesh of two adjacent rollers, and $t_{i}^{\prime}$ is the distance between two adjacent contact points along the plate thickness direction. When the contact point of roller $i$ is above the contact point of roller $i+1, t_{i}$ and $t_{i}^{\prime}$ are positive. When the contact point of roller $i$ is below the contact point of roller $i+1, t_{i}$ and $t_{i}^{\prime}$ are negative. $\theta_{i}$ is the contact angle of roller $i$ with the plate and it is an acute angle. $\theta_{i}$ is positive when it is located in the positive half of the $z$-axis. Otherwise, $\theta_{i}$ is negative.

When the first roller of leveling span $I$ is the upper roller, the following equation can be established:

$$
\left\{\begin{array}{l}
l_{i}^{\prime}=l_{i}-r \theta_{i}-r \theta_{i+1}, \\
t_{i}^{\prime}=t_{i}-r \theta_{i}^{2} / 2-r \theta_{i+1}^{2} / 2 .
\end{array}\right.
$$

When the first roller of leveling span $I$ is the lower roller,

$$
\left\{\begin{array}{l}
l_{i}^{\prime}=l_{i}+r \theta_{i}+r \theta_{i+1} \\
t_{i}^{\prime}=t_{i}+r \theta_{i}^{2} / 2+r \theta_{i+1}^{2} / 2 .
\end{array}\right.
$$

During the leveling process, Eq. (7) can be established for a plate within a leveling span $I$ under the local coordinate system $C S_{I}$ as follows:

$$
\left\{\begin{array}{l}
A_{x}=A_{i}+\Delta A_{x} \\
\tan \theta_{x}=\tan \theta_{i}+\int_{0}^{x} A_{x}\left(1+f^{\prime}(x)^{2}\right)^{3 / 2} \mathrm{~d} x, \\
t_{x}=\int_{0}^{x} \tan \theta_{x} \mathrm{~d} x
\end{array}\right.
$$


where $A_{i}$ is the plate reverse bending curvature at the contact point of roller $I, A_{x}$ is the plate reverse curvature at a distance $x$ from the contact point, $\Delta A_{x}$ is the change in reverse curvature, $\theta_{x}$ is the plate dip angle at a distance $x$ from the contact point, and $t_{x}$ is the plate deflection at a distance $x$ from the contact point.

When $x$ is equal to $l_{i}^{\prime}$, the location is the contact point between the plate and roller $i+1$. As a result, Eq. (7) can be written as:

$$
\left\{\begin{array}{l}
\tan \theta_{i+1}=\tan \theta_{i}+\int_{0}^{l_{i}^{\prime}} A_{x}\left(1+f^{\prime}(x)^{2}\right)^{3 / 2} \mathrm{~d} x \\
t_{i}^{\prime}=\int_{0}^{l_{i}^{\prime}}\left(\tan \theta_{i}+\int_{0}^{x} A_{x}\left(1+f^{\prime}(x)^{2}\right)^{3 / 2} \mathrm{~d} x\right) \mathrm{d} X .
\end{array}\right.
$$

Eq. (8) can be rewritten as

$$
\left\{\begin{array}{c}
\tan \theta_{i+1}=\tan \theta_{i}+A_{i}\left(1+f^{\prime 2}\right)^{3 / 2} l_{i}^{\prime} \\
+\int_{0}^{l_{i}^{\prime}} \Delta A_{x}\left(1+f^{\prime}(x)^{2}\right)^{3 / 2} \mathrm{~d} x \\
\mathrm{t}_{i}^{\prime}=\tan \theta_{i} l_{i}^{\prime}+A_{i}\left(1+f^{\prime 2}\right)^{3 / 2}\left(l^{\prime}\right)^{2} / 2 \\
+\int_{0}^{l_{i}^{\prime}}\left(\int_{0}^{x} \Delta A_{x}\left(1+f^{\prime}(x)^{2}\right)^{3 / 2} \mathrm{~d} x\right) \mathrm{d} X .
\end{array}\right.
$$

$a_{i}$ is defined as the integral value of curvature variation and $b_{i}$ is the double integral value of curvature variation, as described in:

$$
\left\{\begin{array}{l}
a_{i}=\int_{0}^{l_{i}^{\prime}} \Delta A_{x}\left(1+f^{\prime}(x)^{2}\right)^{3 / 2} \mathrm{~d} x \\
b_{i}=\int_{0}^{l_{i}^{\prime}}\left(\int_{0}^{x} \Delta A_{x}\left(1+f^{\prime}(x)^{2}\right)^{3 / 2} \mathrm{~d} x\right) \mathrm{d} X .
\end{array}\right.
$$

Thus, Eq. (9) can be rewritten as:

$$
\left\{\begin{array}{l}
\tan \theta_{i+1}=\tan \theta_{i}+A_{i}\left(1+f_{i}^{\prime}\right)^{3 / 2} l_{i}^{\prime}+a_{i}, \\
t_{i}^{\prime}=\tan \theta_{i} l_{i}^{\prime}+A_{i}\left(1+f_{i}^{\prime}\right)^{3 / 2}\left(l_{i}^{\prime}\right)^{2} / 2+b_{i} .
\end{array}\right.
$$

Eq. (11) can be applied to any leveling span, as the leveling process with $n$ rollers can be divided into $n-1$ leveling spans. Thus, a system of equations containing $2(n-1)$ equations can be built. If $a_{i}$ and $b_{i}$ are given, the independent variables to be identified are the contact angles and reverse curvature of each roller, a total of $2 n$ variables. At the same time, during the plate roller leveling process, the reverse bending curvature $A_{1}$ of the first roller is the initial curvature of the plate, which is a known variable. The curvature of the tail roller $A_{n}$ is the unloading curvature of the reciprocal second roller, which is not an independent variable. Thus, the number of independent unknown variables of equations decreased to $2(n-1)$. According to the above analysis, a certain solution exists for the equations (Eq. (11)) of the geometry model. After $a_{i}$ and $b_{i}$ are calculated, a full representation of the leveling processes will be established, consequently making a full investigation achievable.
Therefore, solving $a_{i}$ and $b_{i}$ is the main purpose of the current investigation into the leveling process.

\subsection{Solution of the Integral Value of Curvature Variation}

In this section, the numerical analytical process of $a_{i}$ and $b_{i}$ is described. To take the biting in and tailing out stages into consideration, the solution is constructed under two cases, namely the contact establishment and dynamic leveling phases. It is widely known that plate reverse bending requires at least three rollers. The leveling process starts when the plate head touches the third roller, which is named as the contact establishment phase. After contact establishment, the plate moves forward with the rotation of the rollers; this is named as the dynamic leveling phase. Correspondingly, the next contact is established when the plate head touches the fourth roller. Thus, the leveling process can be regarded as a process of alternating between contact establishment and dynamic leveling.

\subsubsection{Contact Establishment Phase}

When element 1 touches the nth roller and the element $j_{1}$ touches the first roller, the $n$ roller leveling process is established and it can be described by Eq. (12). The subscript 1 in $j_{1}$ indicates the number of rollers the element touches and $l$ represents the distance between two rollers:

$$
\left\{\begin{array}{l}
n_{1}=l / \Delta x \\
j_{1}=(n-1) n_{1}+1
\end{array}\right.
$$

The first roller in the leveling span $I$ is the roller $i$. When element $j_{i}$ touches the roller $i$, the reverse bending curvature of the element $j_{i}$ can be expressed by $A_{w\left(j_{i}\right)}$. The initial curvature of element $j_{i}$ is $A\left(j_{i}\right)$, which can be obtained by Eq. (3). The relationship in Eq. (13) is constructed for the total bending curvature $A_{j_{i}}$ :

$$
\left\{\begin{array}{l}
j_{i}=j_{1}-(i-1) n_{1} \\
A_{j_{i}}=A_{w\left(j_{i}\right)}-A\left(j_{i}\right) .
\end{array}\right.
$$

The bending moment $M_{j_{i}}$ of element $j_{i}$ can be obtained from its total bending curvature $A_{j_{i}}$, as discussed in Ref. [30]. In the same manner, the total bending curvature $A_{j_{i+1}}$ and bending moment $M_{j_{i+1}}$ of element $j_{i}+1$ that touches the roller $i+1$ can be obtained.

In the leveling span $I$, the bending moment distribution of the plate between two adjacent rollers is linear. As long as $M_{j_{i}}$ and $M_{j_{i+1}}$ are obtained, the bending moment of an arbitrary element $j$ between roller $i$ and roller $i+1$ can be calculated using the following equation: 


$$
M_{j}=M_{j_{i}}+\frac{M_{j_{i+1}}-M_{j_{i}}}{n_{1}}\left(j-j_{i}\right) .
$$

The total bending curvature $A_{j}$ of the element $j$ within the leveling span $I$ can be obtained from its bending moment $M_{j}$, which can be described as follows:

(1) Assuming that the material behaves as an ideal elastic-plastic, the relationship between the curvature and moment of the simple bending process follows Eq. (15), as discussed in Ref. [6]. In the equation, $M_{t}$ is the maximum bending moment of flexible bending and $A_{t}$ is the maximum bending curvature of flexible bending. The inverse function of Eq. (15) can be expressed as Eq. (16). The subscript of $A_{j}^{0}$ represents the number of the calculated element and the superscript represents the number of iterations, as the solving process needs iteration. When the superscript is 0 , the variable is the initial value. The item $\operatorname{sgn}\left(M_{j}\right)$ is to preserve the sign of $M_{j}$.

$$
\begin{aligned}
& M_{j}=M_{t}\left(1.5-0.5 \frac{A_{t}^{2}}{\left(A_{j}^{0}\right)^{2}}\right), \\
& A_{j}^{0}=\operatorname{sgn}\left(M_{j}\right) A_{t} \sqrt{\left(3-2 \frac{M_{j}}{M_{t}}\right)^{-1}} .
\end{aligned}
$$

(2) The bending moment $M_{j}^{0}$ can be calculated according to $A_{j}^{0}$. If $\left|M_{j}^{0}\right|>\left|M_{j}\right|$, the total bending moment $A_{j}$ is between 0 and $A_{j}^{0}$. When the upper and lower bounds of the total curvature are determined, the numerical solution of the total bending curvature in the given error range can be obtained using a dichotomous method. If $\left|M_{j}^{0}\right|<\left|M_{j}\right|, A_{j}^{1}$ is considered as $2 A_{w j}^{0}$. Subsequently, the moment $M_{j}^{1}$ is calculated according to $A_{j}^{1}$. If $\left|M_{j}^{1}\right|>\left|M_{j}\right|$, it indicates that the total bending moment $A_{j}$ is between $A_{j}^{0}$ and $A_{j}^{1}$. The dichotomy method is used to find the numerical solution of $A_{j}$. If $\left|M_{j}^{1}\right|<\left|M_{j}\right|, A_{j}^{2}$ is considered as $3 A_{j}^{0}$. In this case, the numerical solution for the total bending curvature within a given error range can be obtained.

When the total bending curvature $A_{j}$ of element $j$ is obtained, its initial curvature is $A(j)$, which is calculated by Eq. (3). Its reverse bending curvature follows as

$$
A_{w j}=A_{j}-A(j) \text {. }
$$

As the curvature of the element is obtained, the solving steps of its slope are as follows.

(1) Because the moment of element $j_{1}$ that touches the first roller is 0 , its slope does not change during leveling. Its slope $g_{j_{1}}$ can be solved according to Eq. (2). $g_{j_{1}-1}^{0}$, which is the initial slope of element $\left(j_{1}-1\right)$, is initially set as $g_{j_{1}} \cdot g_{j_{1}-1}^{1}$ can be calculated from Eq. (18). If the relative change in the two values is less than the given precision, the iteration stops. If the relative change in the two values is greater than the given precision, $g_{j_{1}-1}^{2}$ is calculated according to $g_{j_{1}-1}^{1}$. The iteration is run until the relative error of $g_{j_{1}-1}^{m-1}$ and $g_{j_{1}-1}^{m}$ is less than the given precision:

$$
\left\{\begin{array}{l}
g_{j_{1}-1}^{m}=g_{j_{1}-1}^{m-1}+A_{w j\left(j_{1}-1\right)}\left(1+\left(g_{j_{1}-1}^{m-1}\right)^{2}\right)^{3 / 2} \Delta x, \\
\vartheta=\left|\left(g_{j_{1}-1}^{m}-g_{j_{1}-1}^{m-1}\right) / g_{j_{1}-1}^{m-1}\right| .
\end{array}\right.
$$

(2) After the slope $g_{j}$ of element $j$ is obtained, $g_{j}$ is used as the initial value of element $j-1$. The slope of element $j-1$ can be calculated according to the above step. The calculation keeps running until the slopes of all the elements are evaluated.

After calculating the slopes and reverse curvatures of all the elements, the first integral value of curvature variation $a_{i}$ and second integral value of curvature variation $b_{i}$ of the leveling interval $I$ are:

$\left\{\begin{aligned} a_{i} & =\sum_{k=1}^{n_{2}}\left[A_{j_{i}-k}\left(1-g_{j_{i}-k}^{2}\right)^{3 / 2}-A_{j_{i}}\left(1-g_{j_{i}}^{2}\right)^{3 / 2}\right] \Delta x, \\ b_{i} & =\sum_{p=1}^{n_{2}}\left\{\begin{array}{c}\sum_{k=1}^{p}\left[A_{j_{i}-k}\left(1-g_{j_{i}-k}^{2}\right)^{3 / 2}-A_{j_{i}}\left(1-g_{j_{i}}^{2}\right)^{3 / 2}\right] \\ +\sum_{k=1}^{p+1}\left[A_{j_{i}-k}\left(1-g_{j_{i}-k}^{2}\right)^{3 / 2}-A_{j_{i}}\left(1-g_{j_{i}}^{2}\right)^{3 / 2}\right]\end{array}\right\} \Delta x^{2} / 2 .\end{aligned}\right.$

The initial reverse bending curvatures and contact angles of leveling rollers are given. The calculated results are used as inputs for iteration, until the relative error of the calculated bending curvatures and input curvatures is less than the given precision. The numerical analysis process of the leveling establishment phase is shown in Figure 3.

\subsubsection{Dynamic Leveling Phase}

After contact is established, the plate moves forward as the leveling rollers rotate. The dynamic leveling process is analyzed according to the length of the plate moving forward. As the plate is discretized into elements whose length is $\Delta x$, the analysis step increment is $u \Delta x$, where $\mathrm{u}$ is a positive integer. The curvature and stress distribution in the previous step will inevitably impact the subsequent leveling process; the curvature and section stress of the elements obtained from the previous step are regarded as the initial state for subsequent computations. The above-mentioned contact establishment phase is taken as the initial state of the dynamic leveling phase. When the plate advances by $u \Delta x$, the numbers of elements whose curvature and section stress change are $1 \sim j_{1}+u$. These elements can be classified into three categories. 


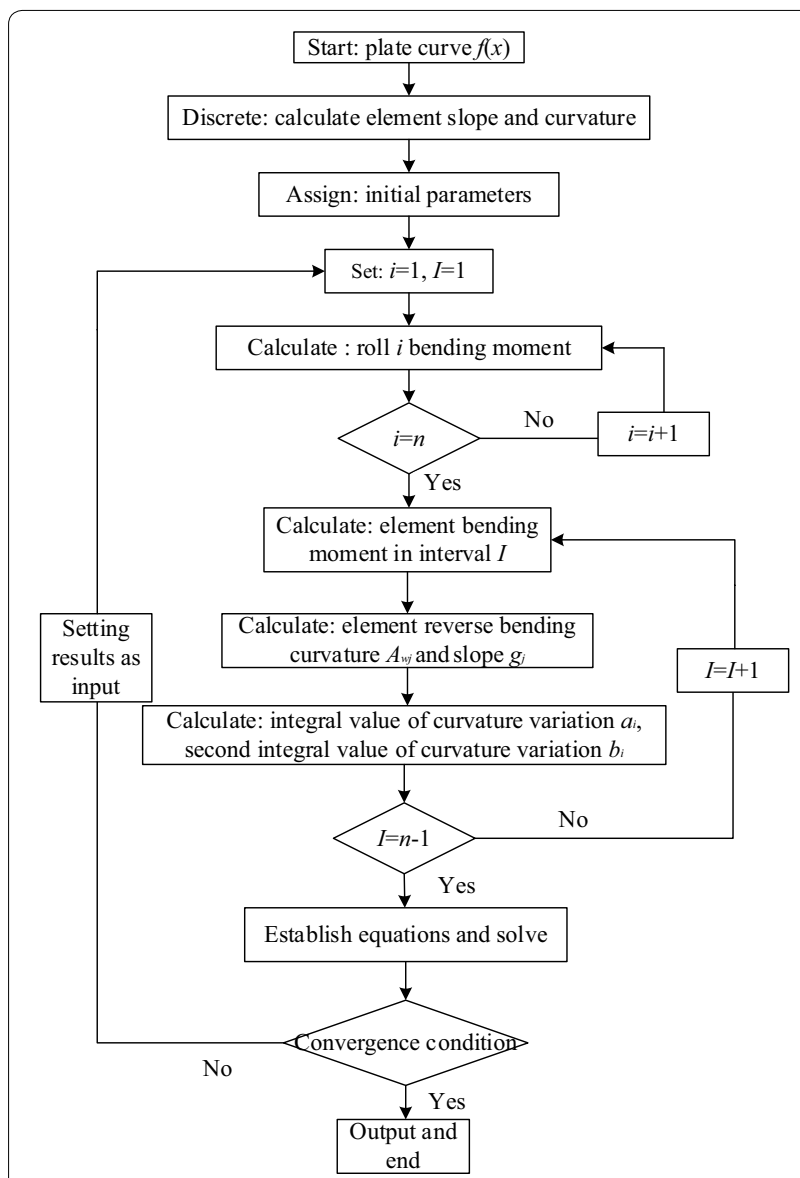

Figure 3 Curvature integration model analysis flow chart in the contact establishment phase

The solutions corresponding to their curvatures are as follows.

The first group consists of elements that move away from the leveling span. The reverse bending effect of the previous analysis step vanishes. Elements $1-u$ are analyzed in this step.

In the preceding analysis step, the bending moment and reverse bending curvature of element $j$ are $M_{j}$ and $A_{w j}$ respectively, which are known quantities. Curvature after rebounding $\left(A_{j}\right)_{2}$ in the current analysis step can be calculated from $M_{j}$ and $A_{w j}$. The subscript 2 of $\left(A_{j}\right)_{2}$ means that the variable represents the current state of the plate and it helps to distinguish from the state of the previous analysis step.

The second group consists of elements entering the leveling span. These elements do not enter the leveling roller originally. During the forward movement of the plate, they enter the leveling span and are bent. These elements numbers are $j_{1}+1$ to $j_{1}+u$ and they are located in the first leveling span.
At this time, the 2nd roller comes into contact with the element whose number is $j_{1}+u-n_{1} \cdot\left(A_{w\left(j_{1}+u-n_{1}\right)}\right)_{2}$ is assigned as the reverse bending curvature of the element $j_{1}+u-n_{1}$. Its initial curvature $\left(A_{\left(j_{1}+u-n_{1}\right)}\right)_{2}$ in the current analysis step is the reverse bending curvature $A_{w\left(j_{1}+u-n_{1}\right)}$ of the previous analysis step. In this way, the total bending curvature $\left(A_{\left(j_{1}+u-n_{1}\right)}\right)_{2}$ can be calculated using Eq. (13). Later, the bending moment $\left(M_{n_{1}+u+1}\right)_{2}$ can be solved from its total bending curvature $\left(A_{\left(j_{1}+u-n_{1}\right)}\right)_{2}$. As the bending moment of the first roller is zero, the bending moment of the elements $j_{1}+1$ to $j_{1}+u$ can be solved using Eq. (14). When the moments of these elements are known, their reverse bending curvatures can be obtained.

The third group consists of elements whose bending moments change with the movement of the plate. These elements are numbered $u+1$ to $j_{1}$.

Firstly, the bending moments of the elements that touch each roller are calculated. Later, the bending moments of the elements $u+1$ to $j_{1}$ are calculated using Eq. (14). For element $j$, whose number is in between $u+1$ and $j_{1}$, the absolute value of the bending moment $\left(M_{j}\right)_{2}$ is subtracted from the absolute value of the bending moment $M_{j}$ of previous analysis step, as shown in Eq. (20). If $M_{j}^{\prime}$ is greater than 0 , it indicates that the element $j$ is in the loading process. If $M_{j}^{\prime}$ is less than 0 , it indicates that the element $j$ is in the unloading process:

$$
M_{j}^{\prime}=\left|\left(M_{j}\right)_{2}\right|-\left|M_{j}\right| .
$$

Assuming that the element $j$ is in the loading process, the increment in bending moment, which is represented by $\Delta M_{j}$, can be calculated as shown in Eq. (21):

$$
\Delta M_{j}=\left(M_{j}\right)_{2}-M_{j}
$$

In this case, the increase in the reverse bending curvature of the element needs to be solved; the corresponding numerical analysis steps are as follows:

(1) Assuming that the material satisfies the ideal elastic-plastic model, a derivative function of Eq. (16) is derived and the curvature increment can be obtained as the initial value, as shown in Eq. (22):

$$
\Delta A_{j}^{0}=A_{t} \sqrt{\left(3-2 \frac{M_{j}}{M_{t}}\right)^{-3}} \Delta M_{j} .
$$

(2) Because the section stress of element $j$ in the previous step is $\sigma_{w j}$, the new section stress $\sigma_{w j}^{0}$ caused by curvature increment $\Delta A_{j}^{0}$ can be calculated. Later, the element moment $\left(M_{j}\right)_{2}^{0}$ is calculated using $\sigma_{w j}^{0}$. If $\left|\left(M_{j}\right)_{2}^{0}-M_{j}\right|>\left|\Delta M_{j}\right|$, it indicates that the curvature increment is between 0 and $\Delta A_{j}^{0}$. The numerical solution of total bending curvature in the given error 
range can be obtained using a dichotomous method. If $\left|\left(M_{j}\right)_{2}^{0}-M_{j}\right|<\left|\Delta M_{j}\right|, \Delta A_{j}^{1}$ is considered as $2 \Delta A_{j}^{0}$. Subsequently, the bending moment $\left(M_{j}\right)_{2}^{1}$ is calculated from $\Delta A_{j}^{1}$. If $\left|\left(M_{j}\right)_{2}^{1}-M_{j}\right|>\left|\Delta M_{j}\right|$, it indicates that the curvature increment is between $\Delta A_{j}^{0}$ and $\Delta A_{j}^{1}$, and the total bending curvature is obtained using a dichotomous method. If $\left|\left(M_{j}\right)_{2}^{1}-M_{j}\right|<\left|\Delta M_{j}\right|, \Delta A_{j}^{2}$ is considered as $3 \triangle A_{j}^{0}$. In this case, the numerical solution for the increment in the reverse bending curvature within a given error range can be obtained.

When the element curvature increment $\Delta A_{j}$ is obtained, its reverse bending curvature satisfies the following equation:

$$
\left(A_{w j}\right)_{2}=A_{w j}+\Delta A_{j} .
$$

For elements in the unloading process, the solution procedures corresponding to the curvature increments are roughly the same as those for the elements in the loading process. The difference lies in the solution of section stress. The section stress for loading elements is given by the superposition of the bending stress and previous section stress. However, the section stress for unloading elements is given by the superposition of the rebounding stress and previous section stress.

The numerical analysis process corresponding to the dynamic leveling phase is shown in Figure 4.

\section{Verification}

In order to evaluate the performance of the proposed model, an experimental study of the plate roller leveling process was conducted. The results obtained from the curvature integration model are compared with the experimental results.

\subsection{An Experimental Plate and Leveling Machine}

A cold-rolled 409L stainless steel plate was selected as the experimental material. Tensile tests were conducted to obtain the material mechanical parameters. The geometrical parameters of the specimen are shown in Figure 5 and the test results are shown in Figure 6. As the reinforcement effect of the material is weak, the material is considered an ideal elastic-plastic material. The Young's modulus was found to be $210 \mathrm{GPa}$, while the yield limit was $245 \mathrm{MPa}$.

The experimental leveling machine is shown in Figure 7 (a). The width of the leveling roller was $500 \mathrm{~mm}$; the leveling speed varied from $0.1 \mathrm{~m} / \mathrm{s}$ to $10 \mathrm{~m} / \mathrm{s}$ and the motor power was $5 \mathrm{~kW}$. To ensure a stable leveling length, only 5 rollers were used during leveling, as the length of the plate was $1000 \mathrm{~mm}$. The arrangement of the 5 rollers is illustrated in Figure 7(b).

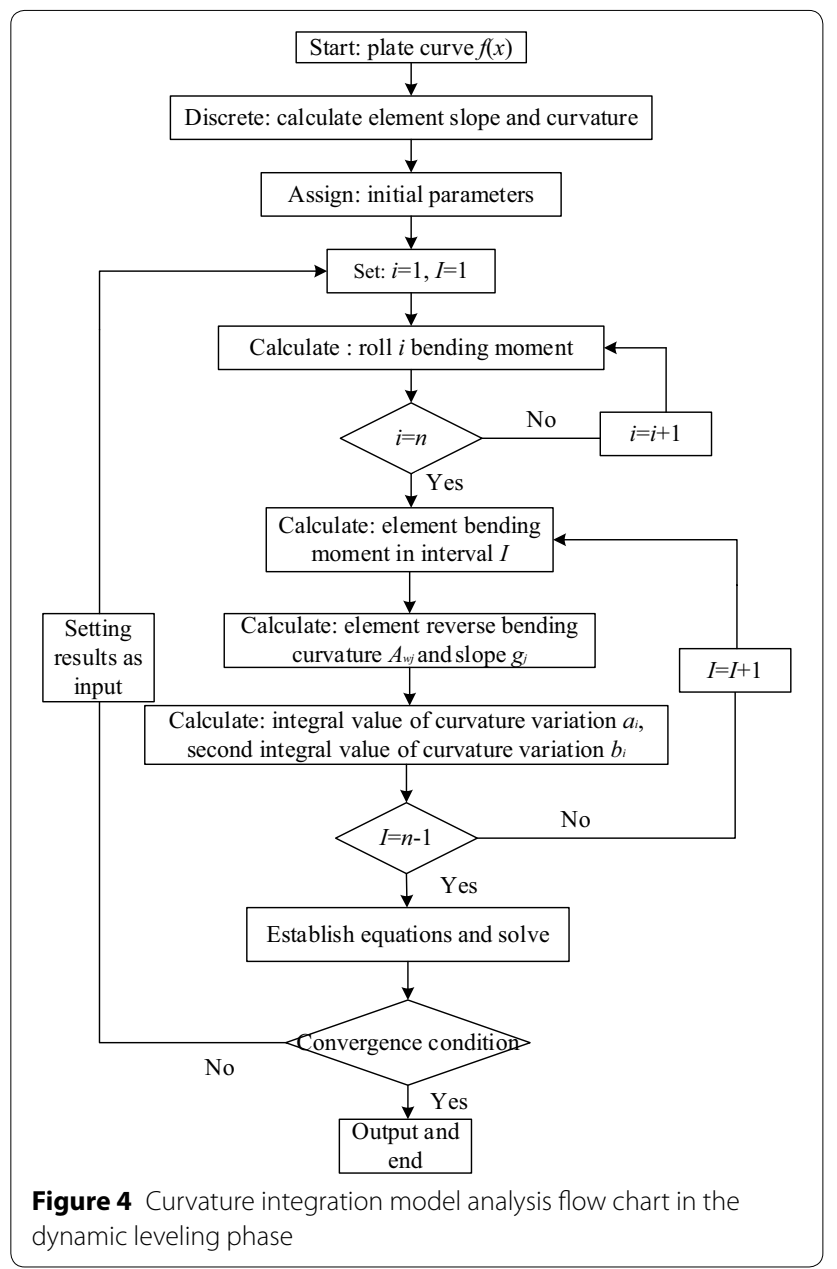

\subsection{Plate Shape Measurement}

The deflection curve of the leveled plate can be used as a key index to evaluate the leveling process. Meanwhile, curvature distribution can be calculated from the plate deflection curve. Therefore, the deflection curve is measured using a 3D laser scanner. It should be mentioned that the plate thickness is nearly constant during the process. Thus, surface topography obtained from the $3 \mathrm{D}$ laser scanner is used to describe plate deformation during leveling. The measurement process is detailed as follows.

(1) Positioning marker arrangement. The positioning markers are glued to the plate surface to be measured, as shown in Figure 8(a). The markers are coated with a special reflective material. They reflect the light emitted by the device and the reflected data is received by same device.

(2) Data acquisition of the plate surface topography. The plate surface is scanned with a laser scanner. The data is transmitted to the computer and a data 


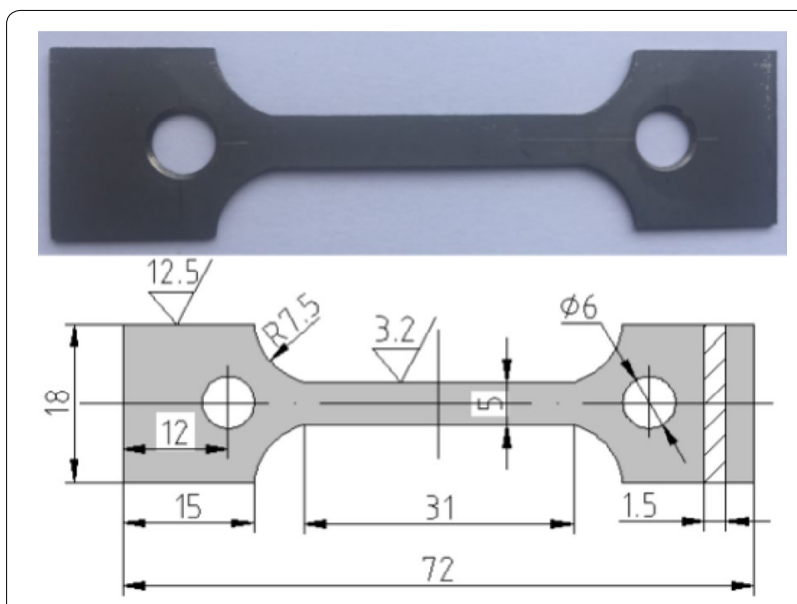

Figure 5 A tensile test specimen

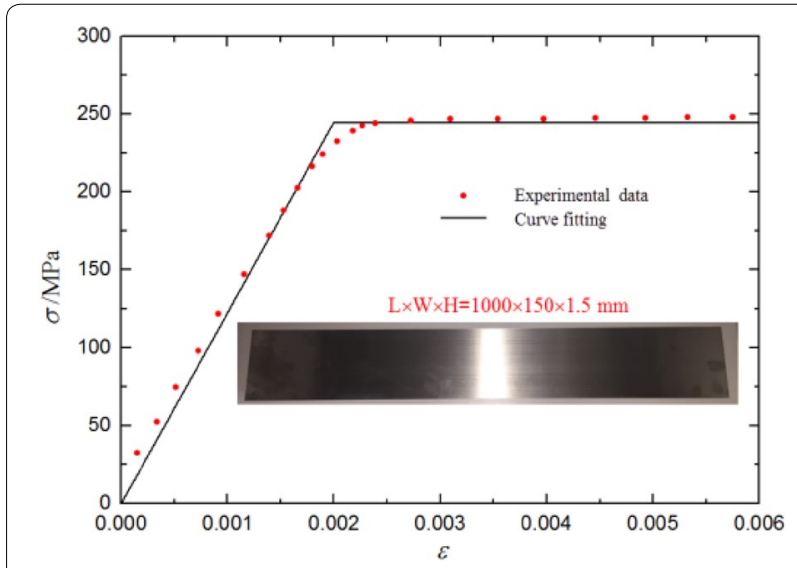

Figure 6 Experimental plate parameters

acquisition software is used for real-time modeling, as shown in Figure 8(b).

(3) Surface model post-processing. The model is imported into the post-processing software Geomagic Studio ${ }^{\mathrm{TM}}$. The holes in the plate model are repaired during the scanning process and the excess parts are removed, as shown in Figure 8(c).

(4) Surface topography model. The post-processing surface topography model is shown in Figure 8(d). The model can be imported into 3D physical modeling software, such as Solidworks ${ }^{\mathrm{TM}}$ and $\operatorname{ProE}^{\mathrm{TM}}$ to obtain further information, such as surface curvature and deflection.

\subsection{Results and Discussion}

Leveling experiments were conducted to evaluate the proposed model. The inter-mesh of roller 2 was $1.5 \mathrm{~mm}$, while that of roller 4 was $0.8 \mathrm{~mm}$. The plate before and after

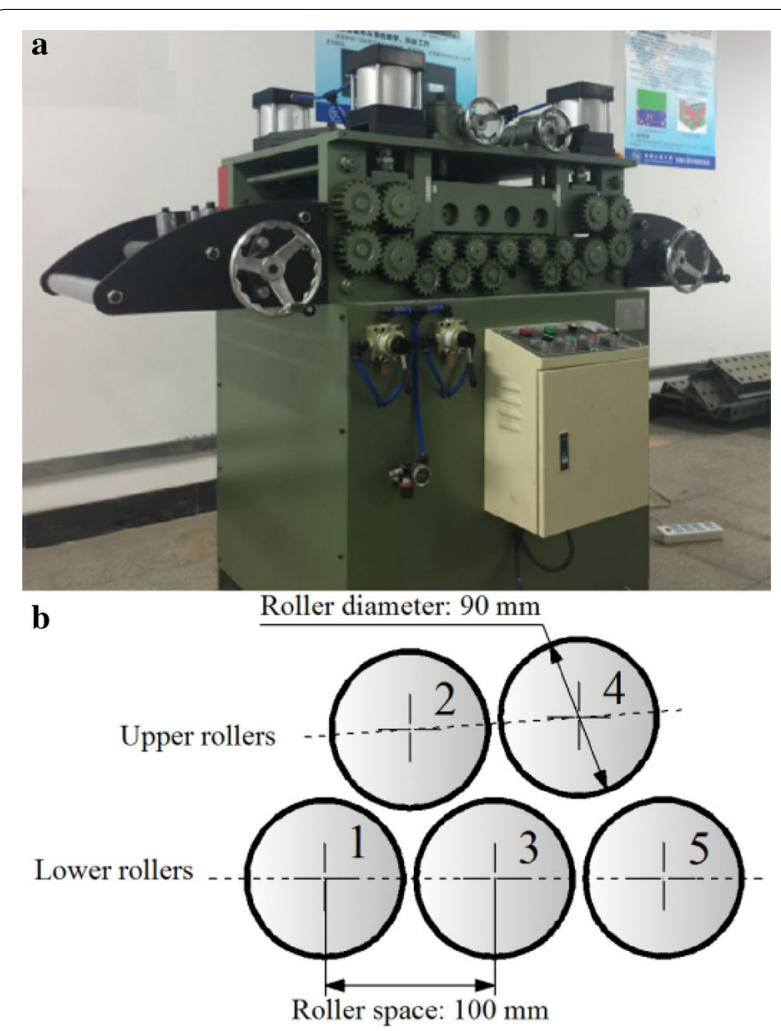

Figure 7 Experimental leveling machine: (a) Leveling machine; (b) Roller arrangement

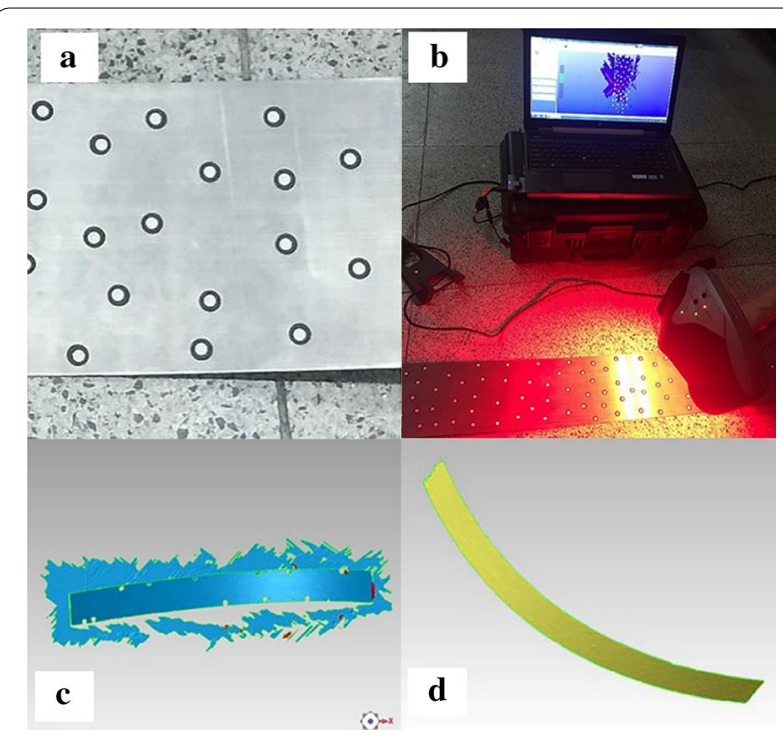

Figure 8 Measurement process: (a) Positioning marker arrangement; (b) Data acquisition site; (c) Model post-processing; (d) Surface topography model 


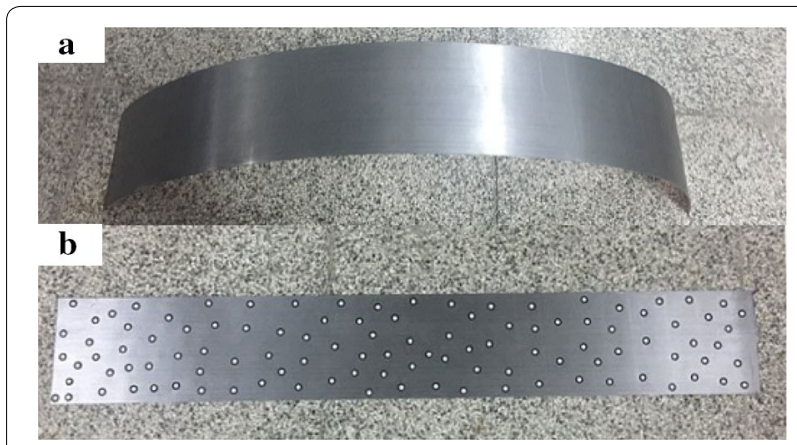

Figure 9 Experimental plate: (a) Before leveling; (b) After leveling

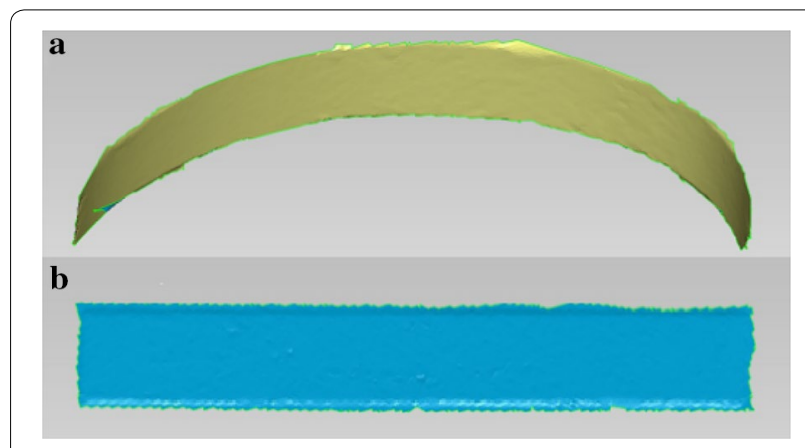

Figure 10 Surface scanning results: (a) Before leveling; (b) After leveling

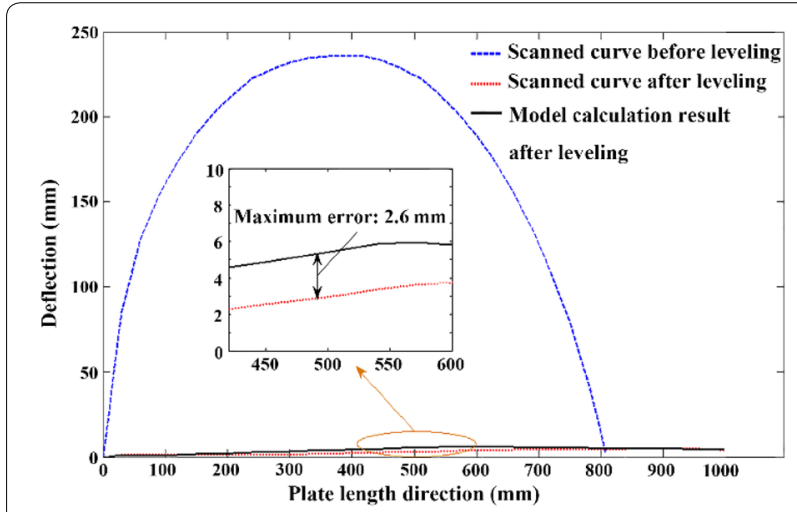

Figure 11 Plate deflection curve

leveling is shown in Figure 9. The surface scanning results are shown in Figure 10. The deflection curve before leveling is extracted along the middle of the plate and fitted with the function shown in Eq. (24):

$$
z=a x^{4}+b x^{3}+c x^{2}+d x+e,
$$

where $\quad a=-4.898 \times 10^{-9}, \quad b=-8.28 \times 10^{-6}$, $c=-5.11 \times 10^{-3}, d=1.844$, and $e=21.16$.

Deflection curves along the length direction of the plate obtained experimentally and by model parsing are shown in Figure 11. The maximum error of the deflection

\section{Table 1 Leveling parameters}

\begin{tabular}{ll}
\hline Parameter & Value \\
\hline Young's modulus (GPa) & 206 \\
Yield stress (MPa) & 245 \\
Plate length (mm) & 1000 \\
Plate thickness (mm) & 1.5 \\
Number of rollers & 5 (top: 2; \\
& bottom: \\
Roller diameter (mm) & 90 \\
Roller space (mm) & 100 \\
Inter-mesh of entrance (mm) & 1.3 \\
Inter-mesh of exit (mm) & 0.8 \\
\hline
\end{tabular}

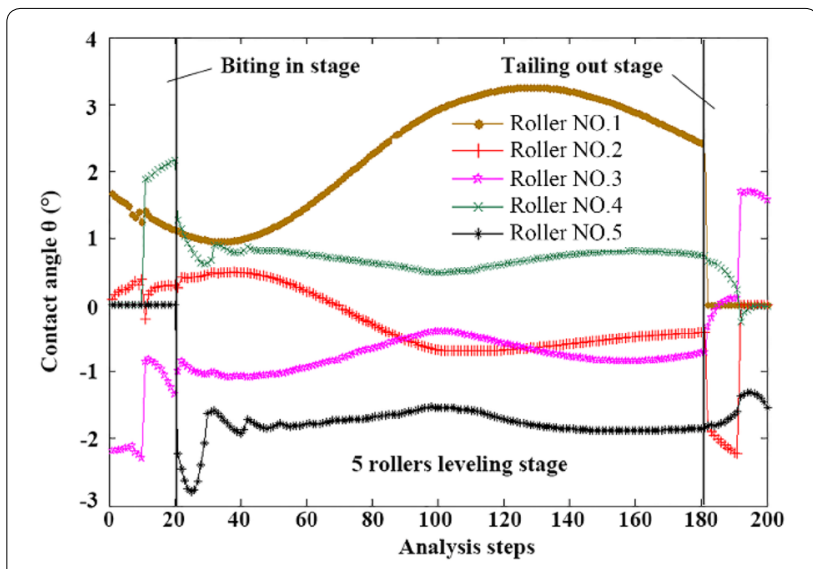

Figure 12 Contact angle analysis

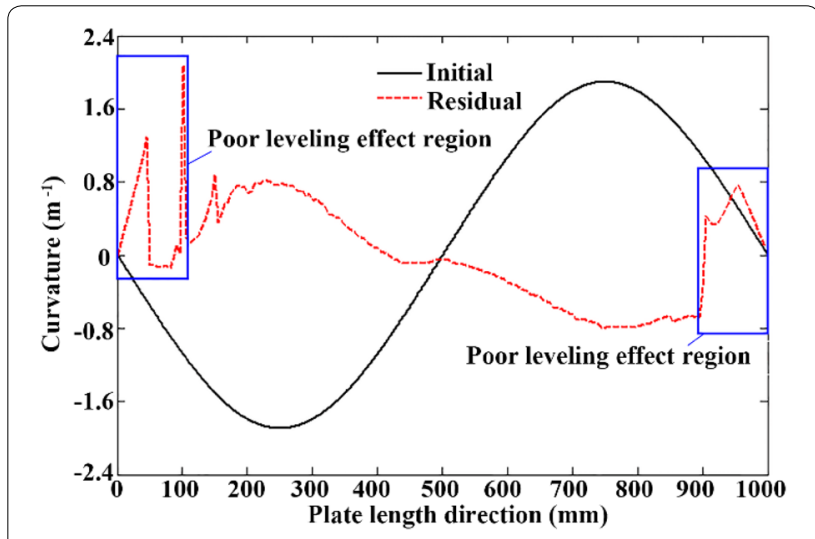

Figure 13 Curvature distribution

curve between experimental and model analyses is 2.6 $\mathrm{mm}$. Because the model calculation results are very close to the experimental results, it can be concluded that the proposed model can predict the plate leveling process effectively. 


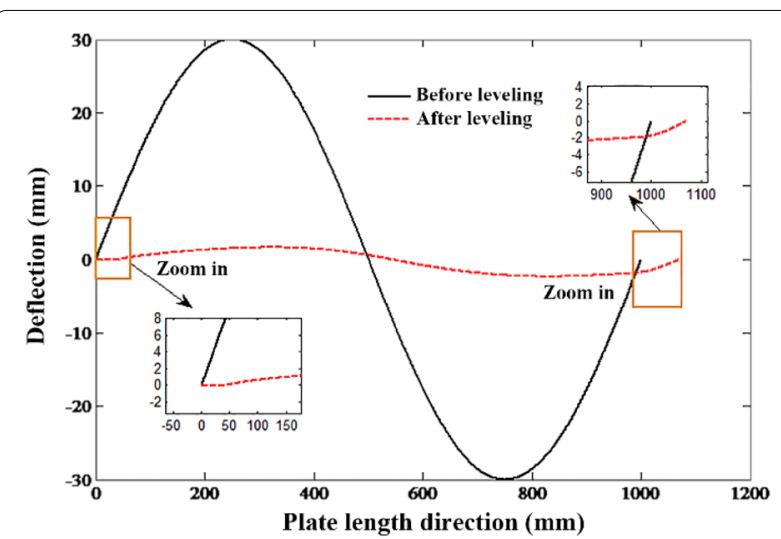

Figure 14 Plate deflection curve

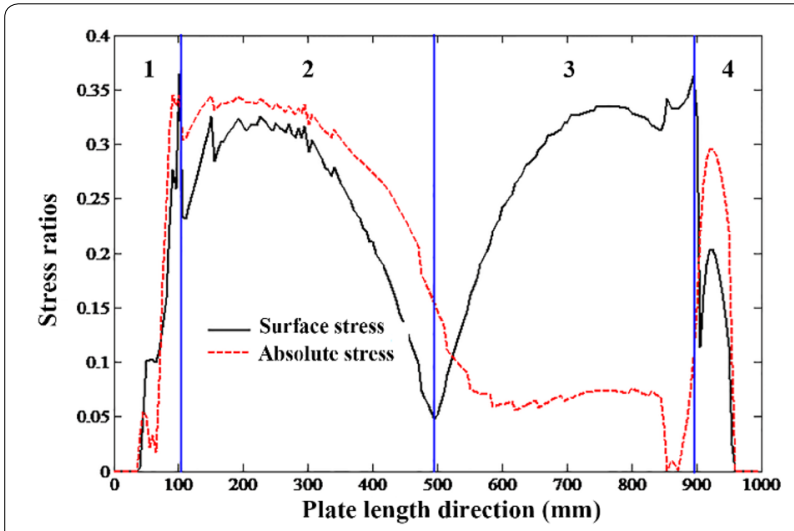

Figure 15 Residual stress

\section{Simulation Results and Discussion}

The proposed model was verified in Section 3. In this section, the model is used to predict the plate leveling process. Taking the plate roller leveling process mentioned above as the research object, the leveling parameters are shown in Table 1. The initial deflection curve function of the plate in question is shown in Eq. (25):

$$
z=30 \sin \frac{\pi x}{500} .
$$

$\Delta x$ is set as $0.5 \mathrm{~mm}$ and $u$ is 10 . The plate goes through 200 analysis steps during the leveling process, which can be divided into 3 stages, namely the biting in stage, the 5 roller leveling stage, and the tailing out stage.

As mentioned earlier, the contact angle has a remarkable impact on plate curvature. Therefore, it is necessary to discuss contact angle values during the leveling process. The plate contact angles of each roller are calculated using the proposed model, as shown in Figure 12. It can be seen that the contact angles vary greatly during the biting in stage and tailing out stage; they are relatively constant except for roller No. 1 during the 5 roller leveling stage. In addition, the contact angle of roller No. 2 is the smallest, which is close to 0 .

Curvature is also a key parameter to study the leveling process and evaluate the leveled plate. Curvature distribution before leveling and after leveling is shown in Figure 13. It can be seen that plate bending is eliminated significantly after the roller leveling process. However, for the head and tail regions of the plate, roller leveling is not effective. That is to say that there are regions at the plate head and end that cannot be leveled by the roller leveling method. The non-leveling region length is nearly $200 \mathrm{~mm}$, about 2 times greater than the roller space.

The plate deflection curve directly reflects the leveling effect. The plate deflection curves before and after leveling are shown in Figure 14. It can be seen from the figure that plate deflection obviously decreased after the leveling process. As the bending curvatures of the head region and tail region are hard to eliminate, the plate shape curve includes warping in these regions.

$\int_{-H / 2}^{H / 2}\left|\sigma_{z}\right| \mathrm{d} z / H \sigma_{s}$ is defined as the absolute stress ratio and $\left|\sigma_{H / 2}\right| / \sigma_{s}$ is defined as the surface stress ratio. The absolute stress ratio and surface stress ratio of the leveled plate are shown in Figure 15. According to the stress distribution, the plate can be divided into 4 regions. Region 1 is the plate head region, region 2 is the plate middle region, which is close to the head, region 3 is the plate middle region close to the tail, and region 4 is the plate tail region. It can be seen from the figure that the residual stress is initially zero and then rapidly increased in region 1 and region 4 of the plate. The maximum value of residual stress is found in region 1 . The theoretical demarcation point of the surface residual stress distribution of region 2 and region 3 is 500 in the length direction. However, the actual demarcation point is less than 500 because of advanced contact, which is responsible for the contact angle. Distribution of the surface residual stress in region 2 and region 3 is basically symmetrical, but the absolute stress in region 2 is far greater than that in region 3.

\section{Conclusions}

(1) The curvature of the plate should be solved precisely and the slope should be calculated to avoid errors in deflection calculation. To take the biting in and tailing out stages into consideration, the proposed curvature integration model is solved in two phases, which include the contact establishment phase and dynamic leveling phase.

(2) A plate leveling experiment was conducted. The proposed model was verified by comparing the 
deflection curves obtained from the proposed model and experiments.

(3) The contact angle varies greatly during the biting in and tailing out stages, but it is mostly relatively constant during the stable leveling stage. The roller leveling process has a significant effect on eliminating plate bending, but it can hardly work in the head and tail regions of the plate.

\section{Authors' Contributions}

$B G, Y Z$ and $C Z$ conceived the model framework; $C Z$ and $B G$ was in charge of the whole trial; $C Z$ and $B G$ wrote the manuscript; $Y W$ assisted with sampling and laboratory analyses. All authors read and approved the final manuscript.

\section{Author Details}

${ }^{1}$ School of Mechanical Engineering, University of Science and Technology Beijing, Beijing 100083, China. ${ }^{2}$ Institute of Artificial Intelligence, University of Science and Technology Beijing, Beijing 100083, China.

\section{Authors' Information}

Ben Guan, born in 1985, is currently an associate professor at School of Mechanical Engineering, University of Science and Technology Beijing, China. He is also a research associate at Institute of Artificial Intelligence, University of Science and Technology Beijing, China.

Chao Zhang, born in 1990, is currently a PhD. He received his philosophy degree from University of Science and Technology Beijing, China.

Yong Zang, born in 1963, is currently a Prof. at School of Mechanical Engineering, University of Science and Technology Beijing, China.

Yuan Wang, born in 1989, is currently a PhD candidate at School of Mechanical Engineering, University of Science and Technology Beijing, China.

\section{Competing Interests}

The authors declare that they have no competing interests.

\section{Funding}

Supported by National Hi-tech Research and Development Program of China (863 Program, Grant No. 2013AA031302), and National Natural Science Foundation of China (Grant No. 51805024).

Received: 27 June 2017 Accepted: 25 April 2019

Published online: 16 May 2019

\section{References}

[1] W Grimm, J Korth, W Kohler. Ilsenburg heavy-plate mill: Modernisation of the mill stand area and of the hot-plate leveler. Iron Steel Review, 2008, 51(9): 11.

[2] V Philippaus, S Mailllard. Modern levelers for advanced plate grade. Iron Steel Review, 2009, 52(10): 144

[3] H Z Xu, K Liu, X H Peng, et al. Research of the image processing in dynamic flatness detection based on improved laser triangular method. Academic Journal of Xi'an Jiaotong University, 2008, 20(3): 168-171.

[4] F Cui. Straightening and straightening machine. 2nd ed. Beijing: Metallurgy Industry Press, 2002. (in Chinese)

[5] A R He, D Z Liu, C Liu. Research on roller straightening mechanical behavior of plastic hardening material. Journal of Mechanical Engineering, 2016, 52(18): 85-91. (in Chinese)

[6] ZY Ma, L F Ma, R J Wang, et al. Study on control strategies of two-roll straightening for bar high precision straightening. Journal of Mechanical Engineering, 2017, 53(20): 77-88. (in Chinese)

[7] Q H Fan, H Zhang, X C Jiang. Leveling theory and experiment study on high strength steel plate of excavator working arm. Journal of Mechanical Engineering, 2017, 53(8): 82-90. (in Chinese)
[8] GCYu, J Zhao, R Ma, et al. Uniform curvature theorem by reciprocating bending and its experimental verification. Journal of Mechanical Engineering, 2016, 52(18): 57-63. (in Chinese)

[9] J Zhao, H Q Cao, P P Zhan, et al. Pure bending equivalent principle for over-bend straightening and its experimental verification. Journal of Mechanical Engineering, 2012, 48(8): 28-33. (in Chinese)

[10] Z Q Zhang, Y H Yan, H L Yang. Research of section deformation of brazier effect in continuous straightening a thin-walled tube. Journal of Mechanical Engineering, 2015, 51(10): 62-68. (in Chinese)

[11] Z Q Zhang, Y H Yan, H L Yang. The straightening curvature-radius model for the thin-walled tube and its validation. Journal of Mechanical Engineering, 2013, 49(21): 160-167. (in Chinese)

[12] Y Zang, H G Wang, F L Cui. Elastic-plasticity analyze of bending deflection on section roller straightening. Journal of Mechanical Engineering, 2005, 41(11): 47-52. (in Chinese)

[13] B Guan, Y Zang, X N Pang, et al. Stress distribution and reverse bending behavior of section during roller leveling process. Journal of Central South University (Science and Technology), 2012, 43(5): 1740-1745.

[14] C L Zhou. Simulation and mathematical model on hot plate roller leveling. Shenyang: Northeastern University, 2006. (in Chinese)

[15] D Y Liu, A R He, H B Wang, et al. Reverse bending research of leveling on plastic hardening material. Journal of Mechanical Engineering, 2015, 51(8): 76-82. (in Chinese)

[16] M Grüber, G Hirt. A strategy for the controlled setting of flatness and residual stress distribution in sheet metals via roller levelling. Procedia Engineering, 2017, 207: 1332-1337.

[17] M Laugwitz, S Seuren, M Jochum, et al. Development of levelling strategies for heavy plates via controlled FE models. Procedia Engineering, 2017, 207: 1349-1354.

[18] H L Gui, Q Li, Q X Huang. The influence of bauschinger effect in straightening process. Mathematical Problems in Engineering, 2015(4): 1-5.

[19] H L Gui, Q Li, Y Li, P Li, Q X Huang. Analysis of rolled piece deformation in straightening process using Fm-Bem. Journal of Marine Science and Technology-Taiwan, 2014(22): 550-556.

[20] Q D Zhang, S Zhou, X F Zhang, et al. Analytic modeling and corroborating by FEM of tension leveling process of thin buckled steel strip. Journal of Mechanical Engineering, 2015, 51(2): 49-57. (in Chinese)

[21] H Huh, HW Lee, R P Sang, et al. The parametric process design of tension levelling with an elasto-plastic finite element method. Journal of Materials Processing Technology, 2001, 113(1): 714-719.

[22] N Mathieu, R Dimitriou, A Parrico, et al. Flatness defects after bridle rolls: a numerical analysis of leveling. International Journal of Material Forming, 2013, 6(2): 255-266

[23] J Yoon. Numerical simulation of continuous tension leveling process of thin strip steel and its application. Journal of Iron and Steel Research, 2007, 14(6): 8-13.

[24] C Betego'n Biempica, J J del Coz Dı́az, P J Garcia Nieto, et al. Nonlinear analysis of residual stresses in a rail manufacturing process by FEM. Applied Mathematical Modelling, 2009, 33: 34-53.

[25] K Kadota, R Maeda. A model of analysis of curvature in leveling processnumeric study of roller leveling process. Jpn. Soc. Technol. Plast., 1993, 34: 481-486.

[26] THigo, H Matsumoto, S Ogawa. Effects of numerical expression of stressstain curve on curvature of material of roller leveling process. Jpn. Soc. Technol. Plast., 2002, 43(496): 439-443.

[27] J A Xue. Theoretical analysis for plate leveling process and its control system. Shenyang: Northeastern University, 2009. (in Chinese)

[28] Z F Liu, Y Q Wang, X C Yan. A new model for the plate leveling process based on curvature integration method. International Journal of Mechanical Sciences, 2012, 54: 213-224.

[29] L Cui, Q Q Shi, X H Liu, et al. Residual curvature of longitudinal profile plate roller in leveling process. Journal of Iron and Steel Research, 2013, 20(10): 23-27.

[30] B Guan, Y Zang, D P Wu, et al. Stress-inheriting behavior of H-beam during roller straightening process. Journal of Materials Processing Technology, 2017, 244: 253-272 